\title{
Polarised and entangled hyperon-antihyperon pairs in BESIII
}

\author{
Karin Schönning for the BESIII collaboration ${ }^{a, *}$ \\ ${ }^{a}$ Dept. of Physics and Astronomy, Uppsala University, Box 516, 75120 Uppsala, Sweden \\ E-mail: karin.schonning@physics.uu.se
}

Polarised and entangled hyperons provide a powerful diagnostic tool to study non-perturbative QCD and to search for physics beyond the Standard Model at the precision frontier. The BESIII experiment in China offer unique possibilities to study strange hyperon-antihyperon final states under particle-antiparticle-symmetric conditions. Recent results include the first complete measurement of the time-like structure of any baryon, as well as a precise CP symmetry test for the $\Lambda$ hyperon. Both analyses exploit the self-analysing weak decay of hyperons, giving access to spin polarization and entanglement of the hyperon-antihyperon pair. Using a similar approach, new information of production and decay of a number of single- and multi-strange as well as charmed hyperons. These proceedings present a summary of the most recent findings and give a glimpse of ongoing studies where new results are expected in the near future.

40th International Conference on High Energy physics - ICHEP2020

July 28 - August 6, 2020

Prague, Czech Republic (virtual meeting)

\footnotetext{
${ }^{*}$ Speaker
} 


\section{Introduction}

The Standard Model of particle physics has proven immensely successful in describing the fundamental particles and their short-range interactions. However, its usefulness has limitations when it comes to describing one of the most abundant building blocks of our visible Universe, i.e. the nucleon. Describing fundamental properties such as the spin [1, 2], structure [3, 4], size [5-7] and abundance [8] remain objects of vital discussions and research. This is largely because the non-perturbative nature of the strong interaction makes quantitative predictions from first principles impossible to perform analytically. Instead, approaches are required such as effective field theories, phenomenological models, or Lattice QCD; research areas where the progress is very tightly connected to experimental data. One way forward is to further refine and intensify the studies of the nucleon itself. Another, complementary way to understand the nucleon better is to make a small change to it and see how it reacts. For instance, we can i) scatter on it (structure and spin studies with e.g. elastic or deep inelastic scattering), ii) excite it (spectroscopy) or iii) replace one of the building blocks. In the following, we focus on iii) and replace one or several of the light quarks in the nucleon with heavier strange or charm quarks [9]. The properties of the resulting hyperons provide a new angle on the nucleon and on non-perturbative QCD.

Ground-state hyperons have an advantage compared to nucleons: their spin properties are experimentally accessible through their weak, parity violating decay. For instance, in the weak, two-body decay of a spin $\frac{1}{2}$ hyperon $Y$ into a spin $\frac{1}{2}$ baryon $B$ and a pseudoscalar meson $M$, the angular distribution of the daughter baryon is given by

$$
I\left(\theta_{B}\right) \propto 1+\alpha_{Y} P \cos \theta_{B}
$$

where $\alpha_{Y}$ is the decay asymmetry parameter [10] and $P$ the polarisation. Sequentially decaying hyperons, e.g. $\Xi$ and $\Omega$, provide information on the additional decay parameters $\beta_{Y}, \gamma_{Y}$ and/or $\phi_{Y}$. In this sense, hyperons reveal more information about themselves than nucleons as we will demonstrate in this presentation. Furthermore, by going even deeper we will show how polarised and entangled hyperon-antihyperon pairs from $e^{+} e^{-}$annihilations can provide new insights on two of the most challenging problems in contemporary physics: non-perturbative strong interactions and the matter-antimatter asymmetry of the Universe. New techniques have been developed and are being applied at the BEijing Spectrometer (BESIII) experiment in China. In these proceedings, we will report on the most recent results and summarise the future prospects for hyperon-antihyperon studies at BESIII.

\section{The BESIII experiment}

The BES III detector is an integrated part of the BEPC-II (Beijing Electron Positron Collider), a double-ring $e^{+} e^{-}$collider optimised for a luminosity of $10^{33} \mathrm{~cm}^{-2} \mathrm{~s}^{-1}$ at $\sqrt{s}=3.770 \mathrm{GeV}$ [11]. The BES III detector covers $93 \%$ of the full solid angle and has five components: i) a multiwire drift chamber for tracking, ii) an electromagnetic calorimeter for energy measurement and identification of electrons, positrons and photons, iii) a time-of-flight system, iv) a super-conducting magnet for deflection of particle trajectories, and v) a muon detection system. For more details, see Ref. [12]. BESIII is famous for the discovery of the first confirmed charged charmonium state, the $Z_{c}(3900)^{ \pm}$, with a minimum quark content of $q q \bar{q} \bar{q}$ [13]. 


\section{Recent results}

In $e^{+} e^{-}$colliders, hyperon-antihyperon pairs can be produced either electromagnetically, via an intermediate virtual photon, or from the decay of a vector resonance e.g. charmonium states. In both cases, interference between different production amplitudes result in hyperons that are polarised with respect to the production plane, even if the initial $e^{+} e^{-}$state is unpolarised [14]. Electromagnetic production gives access to the inner structure of the produced hyperon, whereas production through charmonium resonances such as $J / \Psi$ or $\Psi(2 S)$ typically results in large data samples which enables precision studies of decay parameters. The joint angular distributions of entangled hyperon-antihyperon pairs and their subsequent decays can be parameterised in terms of time-like hadronic and electromagnetic form factors and decay asymmetry parameters [15-17].

\subsection{Hyperon structure}

Hadron structure can be quantified by electromagnetic form factors (EMFFs), analytic functions of the momentum transfer squared $q^{2}$. The EMFFs are either space-like $\left(q^{2}<0\right)$ or time-like $\left(q^{2}>0\right)$. The space-like EMFFs are related to charge- and magnetisation densities of the hadron and are studied in elastic lepton-hadron scattering. However, the space-like region is difficult to study for unstable hadrons such as hyperons. Instead, the time-like region, probed in e.g. $e^{+} e^{-} \rightarrow Y \bar{Y}$ processes, provide a viable option. The space-like and the time-like region are related by dispersion relations [18]. It is interesting to note that whereas space-like EMFFs are real, the time-like ones can be complex with a relative phase $\Delta \Phi$. This phase is a quantifies the interference between the production amplitudes and therefore induces a polarising effect on the produced hyperons. However, as $\left|q^{2}\right| \rightarrow \infty$, the space-like and time-like EMFFs should converge to the same value, meaning that $\Delta \Phi$ must be an integer multiple of $\pi$. Hence, the experimentally accessible polarisation of hyperons makes it possible to study the onset of the scale of the space-like/time-like convergence without direct measurements in the space-like region.

The BESIII experiment has recently performed the first complete measurement of the time-like structure of any baryon, through a study of the $\Lambda$ hyperon in the $e^{+} e^{-} \rightarrow \gamma^{*} \rightarrow \Lambda \bar{\Lambda}$ reaction at $2.396 \mathrm{GeV}$. An sample of $\approx 550$ exclusively reconstructed $e^{+} e^{-} \rightarrow \gamma^{*} \rightarrow \Lambda \bar{\Lambda}, \Lambda \rightarrow p \pi^{-}, \bar{\Lambda} \rightarrow \bar{p} \pi^{+}$ events was analysed using the formalism outlined in Ref. [16]. The ratio between the electric and the magnetic form factors, $R=G_{E} / G_{M}$, was measured to be $R=0.96 \pm 0.14$ (stat.) \pm 0.02 (sys.) and the phase was found to be $\Delta \Phi=37^{\circ} \pm 12^{\circ}$ (stat.) $\pm 6^{\circ}$ (sys.) [19]. These results have already sparked progress in the theory community, with interpretations in terms of $\Lambda \bar{\Lambda}$ potential models [20], vector dominance [21] and dispersion theory [22].

The next step is to study the energy dependence of the phase. BESIII has collected an unprecedented sample of off-resonance data between $2.0 \mathrm{GeV}$ and $4.7 \mathrm{GeV}$ that open up new possibilities not only for further studies of the $\Lambda$ hyperon, but also for other octet hyperons such as the $\Sigma$ isotriplet and the $\Xi$ isodoublet, as well as the decuplet $\Omega^{-}$and the charmed $\Lambda_{c}^{+}$.

\subsection{Hyperon decays}

One of the most puzzling, unresolved conundrums of modern science is why the Universe consists of so much more matter than antimatter. Unless this was fine-tuned in the Big Bang, this matterantimatter asymmetry must have a dynamical origin, Baryogenesis [8]. This, however, requires 
processes that violate conservation of fundamental quantum numbers such as charge conjugation and parity (CP). In short, CP symmetry implies that particles and antiparticles have the same decay patterns. In two-body decays of entangled hyperons and antihyperons, differences in decay patterns can be defined in terms of the decay asymmetry parameter $\alpha_{Y}$ : $\mathrm{CP}$ violation would result in a non-zero value of the asymmetry

$$
A_{C P}=\frac{\alpha_{Y}+\alpha_{\bar{Y}}}{\alpha_{Y}+\alpha_{\bar{Y}}} .
$$

A recent work by BESIII, exploiting polarised and entangled $\Lambda \bar{\Lambda}$ pairs from $J / \Psi$ decays, provided the most precise measurement of $A_{C P}$ so far: $0.006 \pm 0.012$ (stat.) \pm 0.007 (syst.) [23]. More importantly, the decay asymmetry $\alpha_{\Lambda}$ was found to be $0.750 \pm 0.009$ (stat.) \pm 0.004 (syst.), in strong disagreement $(\approx 12 \sigma)$ with previous measurements based on the proton polarimeter technique [24]. This triggered a re-analysis of CLAS data that resulted in an $\alpha_{\Lambda}$ that was closer to, though still not consistent $(\approx 3 \sigma)$ with, the BESIII result [25]. Since many measurements in various fields depend directly $\alpha_{\Lambda}, e . g$. hyperon spin observables, spectroscopy, decay parameters of heavier hyperons and beams of polarised protons from $\Lambda$ decays, it is important to understand why the different techniques yield different results. A recently collected world-record sample of $10^{10} \mathrm{~J} / \Psi$ events from BESIII can help resolving this issue since it allows for a more precise determination of systematic effects.

The technique applied in the BESIII $J / \Psi \rightarrow \Lambda \bar{\Lambda}$ measurement was utilized in the analysis of $\Sigma^{+} \bar{\Sigma}^{-}$pairs from $J / \Psi$ and $\Psi(2 S)$ decays. The decay asymmetry $A_{C P}$ was extracted for the first time for the $\Sigma^{+}$and found to be $0.004 \pm 0.037$ (stat.) \pm 0.010 (syst.). In addition, it was found that the hadronic form factor phase $\Delta \Phi$ changes sign between $J / \Psi$ and $\Psi(2 S)$ [26].

So far, all existing measurements of the asymmetry $A_{C P}$ from two-body hyperon decays have been found to be consistent with zero. However, since hyperons decay via an interplay between strong and weak (or possibly even beyond SM) interactions, it is difficult to quantify the sensitivity of $A_{C P}$ to $\mathrm{CP}$ violation. On the one hand, strong CP conserving processes could dilute or hide CP violating components from weak or BSM processes. On the other hand, $A_{C P}$ is to the first order a product of strong and weak phase differences $[27,28]$. Hence, a very small strong phase would result in a vanishing $A_{C P}$ even in the presence of $\mathrm{CP}$ violating processes. Sequentially decaying hyperons, such as the $\Xi$ isodoublet, offer a way to directly measure and separate strong and weak phase differences. This is being utilized in an ongoing BESIII study of polarised and entangled $\Xi^{-} \bar{\Xi}^{+}$pairs, employing the formalism outlined in Refs. [17, 29]. Furthermore, electromagnetic decays of polarised $\Sigma^{0}$ hyperons provide a test for strong CP violation [30]. The formalism for $e^{+} e^{-} \rightarrow \Sigma^{0} \bar{\Sigma}^{0}$ is derived in Ref. [31,32] and is being applied in an ongoing study with BESIII.

Presently, the data samples at BESIII do not allow for exclusive reconstruction of pairs of triple-strange $\Omega^{-} \bar{\Omega}^{+}$or the charmed $\Lambda_{c}^{+} \bar{\Lambda}_{c}^{-}$. However, the joint angular distribution of single-tagged sequential decays of these hyperons provide information on the decay parameters $\alpha_{Y}, \beta_{Y}, \gamma_{Y}$ and/or $\phi_{Y}$. In Ref. [33], the decay asymmetry parameter $\alpha_{\Lambda_{c}^{+}}$was studied for the decay modes $p K_{s}, \Sigma^{0} \pi^{+}$ for the first time. In addition, the precision was improved for the modes $\Lambda \pi^{+}$and $\Sigma^{+} \pi^{0}$. Finally, $\beta_{\Lambda_{c}^{+}}$and $\gamma_{\Lambda_{c}^{+}}$of the modes $\Sigma^{0} \pi^{+}, \Lambda \pi^{+}$and $\Sigma^{+} \pi^{0}$ were determined. A new, large-scale data taking campaign in the region 4.6-4.7 GeV offers increased precision and potentially also a separation of hyperon and antihyperon decay asymmetries. 
The production of spin $\frac{3}{2}$ baryon pairs is described by four complex form factors, or helicity amplitudes, in contrast to two in the case of spin $\frac{1}{2}$ pairs. The formalism for the $e^{+} e^{-} \rightarrow \gamma^{*} / \Psi \rightarrow$ $\Omega^{-} \bar{\Omega}^{+}$, as outlined in Ref. [17], expresses these amplitudes in terms of the seven polarisation parameters $r_{m u}$ that are non-zero for an unpolarised initial state. Applying the aforementioned formalism on the $\Psi$ (3686) data sample collected by BESIII, the helicity amplitudes as well as the decay parameter $\phi_{\Omega}$ were reconstructed. In addition, the degree of polarisation $d\left(\rho_{3 / 2}\right)$ could be calculated using

$$
d\left(\rho_{3 / 2}\right)=\sqrt{\sum_{\mu=1}^{15} \frac{r_{\mu}}{r_{0}}}
$$

The results show a significant polarisation of the $\Omega^{-}$hyperon, where the main contributions come from higher order tensor polarisation effects [34]. The fact that spin $\frac{3}{2}$ baryons can be produced polarised at the kinematic threshold whereas spin $\frac{1}{2}$ cannot, makes baryon-antibaryon pair production in $e^{+} e^{-}$and charmonium decays a suitable testing ground for the baryon spin [17]. This is valuable since the spin of the $\Omega^{-}$hyperon has not yet been determined in a model-independent way [24].

\section{Summary}

Hyperons provide a powerful diagnostic tool to study the strong interaction and fundamental symmetries. In particular, exclusive measurements of polarised and entangled hyperon-antihyperon pairs give access to information that is difficult or impossible to study in other processes. In recent studies by the BESIII collaboration, the structure and decay of the single-strange $\Lambda$ hyperon has been studied with unprecedented precision. Furthermore, in ongoing studies of sequentially decaying multi-strange and charmed hyperons, strong and weak/beyond SM observables can be disentangled. This will, in combination with the world-record data sample of $10^{10} \mathrm{~J} / \Psi$ events from BESIII, has potential to bring hyperon physics to a new level.

\section{Acknowledgement}

The speaker is grateful to the Knut and Alice Wallenberg Foundation, the Swedish Research Council and the Swedish Foundation for International Cooperation in Research and Education.

\section{References}

[1] C. A. Aidala et al., Rev. Mod. Phys. 85, 655 (2013).

[2] C. Alexandrou et al., Phys. Rev. Lett. 119, 142002 (2017).

[3] G. A. Miller, Phys. Rev. Lett. 99, 112001 (2007).

[4] M. Ablikim et al., Phys. Rev. Lett. 124, 042001 (2020).

[5] R. Pohl et al., Nature 466 (2010) 213; C. E. Carlson, Prog. Part. Nucl. Phys. 82, 59 (2015).

[6] N. Bezginov et al., Science 365, Issue 6457, 1007-1012 (2019). 
[7] H.-W. Hammer and U-G. Meissner, Sci.Bull. 65, 257-258 (2020).

[8] A. D. Sakharov, Pisma Zh. Eksp. Teor. Phys. Fiz. 5, 32 (1967).

[9] C. Granados, S. Leupold and E. Perotti, Eur. Phys. J. A 53, 17 (2017).

[10] T. D. Lee and C. N. Yang, Phys. Rev. 108, 1645 (1957).

[11] F. A. Harris et al., Int. J. Mod. Phys. A 24, 377 (2009).

[12] M. Ablikim et al. (BES III Collaboration), Nucl. Instrum. Meth. A 614, 345 (2010).

[13] M. Ablikim et al. (BES III Collaboration), Phys. Rev. Lett. 110, 252001 (2013).

[14] A. Z. Dubnickova et al., Nuovo Cim. A 109241 (1996).

[15] G. Fäldt, Eur. Phys. J. A 52, 141 (2016)

[16] G. Fäldt and A. Kupsc, Phys. Lett. B 772, 16 (2017).

[17] E. perotti et al., Phys. Rev. D 99, 056008 (2019).

[18] M. A. Belushkin et al., Phys. Rev. C 75, 035202 (2007).

[19] M. Ablikim et al., Phys. Rev. Lett. 123, 122003 (2019).

[20] J. Haidenbauer and U.-G. Meissner, Phys. Lett. B 761, 456-461 (2016).

[21] Y. Yang, D.-Y. Chen and Z. Lu, Phys. Rev. D 100, 073007 (2019).

[22] S. Pacetti, talk at the Workshop on Baryon Production at BESIII, USTC Hefei, China (2019).

[23] M. Ablikim et al.,, Nature Phys. 15, 631 (2019).

[24] P. A. Zyla et al. (Particle Data Group), Prog. Theor. Exp. Phys. 2020, 083 C01 (2020).

[25] D. Ireland et al., Phys. Rev. Lett. textbf123, 182301 (2019).

[26] M. Ablikim et al., Phys. Rev. Lett. 125052004 (2020).

[27] J. F. Donoghue, X. G. He and S. Pakvasa, Phys. Rev. D 34, 833 (1986).

[28] J.Tandean andG. Valencia, Phys. Rev. D 67, 056001 (2003).

[29] P. Adlarson and A. Kupsc, Phys. Rev. D 100, 114005 (2019).

[30] S. Nair et al., Phys. Lett. B 788, 535 (2019).

[31] G. Fäldt and K. Schonning, Phys. Rev. D 101, 033002 (2020).

[32] G. Fäldt, Remarks on the analysis of the reaction $e^{+} e^{-} \rightarrow \Sigma^{0} \bar{\Sigma}^{0}$,hep-ph:2005.10689, (2020).

[33] BESIII Collaboration, Phys. Rev. D 100, 072004 (2019).

[34] M. Ablikim et al., Helicity Amplitude Analysis of $\Psi(3686) \rightarrow \Omega^{-} \bar{\Omega}^{+}$, hep-ex:2007.03679, (2020). 